Article

\title{
A Robust Automated Cataract Detection Algorithm Using Diagnostic Opinion Based Parameter Thresholding for Telemedicine Application
}

\author{
Shashwat Pathak * and Basant Kumar \\ Department of Electronics and Communication Engineering, Motilal Nehru National Institute of Technology, \\ Allahabad 211004, India; singhbasant@mnnit.ac.in \\ * Correspondence: shashwat@mnnit.ac.in; Tel.: +91-737-687-8312 \\ Academic Editor: Mostafa Bassiouni \\ Received: 29 June 2016; Accepted: 6 September 2016; Published: 15 September 2016
}

\begin{abstract}
This paper proposes and evaluates an algorithm to automatically detect the cataracts from color images in adult human subjects. Currently, methods available for cataract detection are based on the use of either fundus camera or Digital Single-Lens Reflex (DSLR) camera; both are very expensive. The main motive behind this work is to develop an inexpensive, robust and convenient algorithm which in conjugation with suitable devices will be able to diagnose the presence of cataract from the true color images of an eye. An algorithm is proposed for cataract screening based on texture features: uniformity, intensity and standard deviation. These features are first computed and mapped with diagnostic opinion by the eye expert to define the basic threshold of screening system and later tested on real subjects in an eye clinic. Finally, a tele-ophthamology model using our proposed system has been suggested, which confirms the telemedicine application of the proposed system.
\end{abstract}

Keywords: pupil; cataract; texture information; K-Means clustering; graphic user interface (GUI); MATLAB; telemedicine

\section{Introduction}

Eye related disorders and subsequent vision loss affects individuals severely through on-going medical expenses during treatment of disorders and through the high economic and mental trauma of degrading vision [1]. The World Health Report regarding cataracts, updated in 2014, says that 285 million people are estimated to be visually impaired worldwide, out of those 39 million are blind and 246 have low vision [2]. In the current scenario, cases of cataracts leading to blindness are likely to advance due to an ageing population and shortage of required healthcare infrastructure in low and middle-income countries [3]. World Health Organization (WHO) defines a cataract as clouding of the lens of the eye, which impedes the passage of light [4]. Normally, the lens is a completely clear image. The proteins which form the lens are normally very precisely layered and arranged and it is this feature which allows the proteins to be completely transparent. If this internal structure is degraded and the proteins become disorganized or damaged, the lens itself starts to become whitish or cloudy and this prevents the transmission of light back onto the retina. Depending upon cataract occurrence in the eye we have developed a classification of cataracts which classifies the images into: Nuclear, Cortical and Post Subcapsular Cataract (PSC). There is also another classification based on severity of the cataract affecting the eye, which further classifies the optical eye images into normal, mild, medium and severe cataract. In India, cataract formation is more commonly observed among adult subjects. Today, ophthalmologists use a slit lamp or ophthalmoscope to diagnose cataracts. This equipment is quite expensive and requires special training for use. Due to advancements in digital image processing, diagnosis of eye related diseases can also be made by extraction and identifying 
the significant features from an eye image; e.g., cataracts can be detected by identifying the clouding in the pupil region using different image parameters. Some of the related works are mentioned here. Retno Supriyanti et al. [5,6] proposed a new approach to detect the presence of cataracts based on digital image processing. The authors proposed the use of specular reflection analysis for cataract detection. Retno Supriyanti et al. [7] proposed the necessary specifications and guidelines to detect the presence of cataracts. They performed an effective experiment on a great number of subjects by changing the positions of the camera and subject. Hualei Shen et al. [8] proposed a machine-aided grade classification method for cataractous lens based on color information with different hardness degrees. Recognition rate of 92.5\% was achieved. Meimei Yang et al. [9] worked on detection of parameters for the diagnosis of cataract through retinal images. They used a neural network classifier for automatic cataract detection based on the classification of retinal images. Jagadish Nayak [10] proposed the use of image processing techniques to extract the features of optical eye images and to classify them such as normal, cataract and post-cataract images. The extracted features of the optical eye image were used in the automatic classifier such as Support Vector Machines (SVM) for the automatic classification. Huiqi Li et al. [11] discussed a computer diagnosis system for nuclear cataract detection. This work is based on the analysis of retinal images. Caixinha et al. [12,13] acquired ultrasound A-scan signals from healthy and cataractous porcine lenses, later B-mode images were reconstructed from the collected signals. Nakagami images were constructed from those B-mode images. Different parameters were obtained from the central region of the lens. Image textural parameters were extracted from the B-scan and Nakagami images. The obtained parameters were subjected to feature selection with Principal Component Analysis (PCA) and used for classification through a multiclass Support Vector Machine (SVM). R. Srivastava et al. [14] presented preprocessing, feature extraction and grade prediction of nuclear cataract (NC) from slit-lamp images using image gradients. The lens region was localized automatically using horizontal and vertical profile clustering and then the structure was detected using a modified version of active shape model (ASM). Features such as Location, orientation, and magnitude were extracted from this image. These features are used in combination with prior features related to color and brightness. Guo et al. [15] presented fundus image analysis based automatic classification and grading of cataract. The system was built up with three main stages: preprocessing of image, feature extraction, cataract classification and grading. Features extraction was done using wavelet transform (Haar transform) and a sketch based method combined with discrete cosine transform. To decide between cataract and non-cataract images, multiclass discriminant analysis is used then graded into mild, moderate and severe cataract. The correct classification rate for hybrid of wavelet transform and sketch based method classification and grading is $89.3 \%$ and $73.8 \%$ respectively.

All the discussed works related to computer aided cataract detection use retinal, ultrasound or slit lamp images. These systems have increased complexities, and the cost of acquisition module is very high. These devices require a trained technician for its operation. Development of a low cost system can be accomplished by using digital camera images. These devices are very common and images acquired by them need just a few instructions to be followed to be used in a system developed to detect cataracts based on these true color images. The main limitation of these discussed systems lies in determining the accurate thresholds value of multiple texture parameters such as uniformity, standard deviation and mean intensity of pupil area in digital eye image. This requires exhaustive training and validation of the developed detection algorithms using diagnostic opinion from an ophthalmologist. Further, other major limitations include complexity, ease of use and accuracy of decision. Therefore, an attempt has been made through the present work to develop a robust cataract detection algorithm based on digital color images. Here the decision regarding the presence of the cataract is made by considering three texture features, namely mean intensity, uniformity and standard deviation. Analysis of three features mapped with the diagnostic opinion of an eye expert adds robustness to the proposed algorithm. 
Section 2 presents the description of diagnostic opinion based robust algorithm for automated cataract detection. Results and discussion have been given in Section 3. Finally, Section 4 concludes the overall work with inclusion of future work.

\section{Robust and Efficient Automated Cataract Detection Algorithm}

For the automatic detection of cataract from a digital eye image, extraction of the pupil from the image is required. The pupil of an eye has the same color in all humans, only the iris color differs from person to person. The pupil is circular in shape, so we can easily detect it using common circular region detection algorithms for which Hough transform is the most common choice. It is used here to automatically detect the circular pupil, which is distinguishable. The basic method proposed in this paper for robust cataract detection algorithm can be described in three steps: preprocessing, feature extraction, and decision making.

Preprocessing includes conservative smoothing followed by image denoising. The isotropic Gaussian filter is widely used as a low-pass filter for image de-noising [16]. A two dimensional (2D) Gaussian function is simply the product of two 1D Gaussian functions (one for each direction: horizontal and vertical) and is given by:

$$
G(x, y)=\frac{1}{2 \pi \sigma^{2}} e^{\frac{x^{2}+y^{2}}{2 \sigma^{2}}}
$$

where ' $\sigma$ ' is the standard deviation of the distribution and its value has been chosen as 0.5 in this work. After denoising, edge information will be lost hence it is necessary to preserve the edge. The most common and widely used approach for edge detection is canny edge detection. The smoothened image is then filtered with a Sobel kernel in both horizontal and vertical direction to get the first derivative $(G x)$ and $(G y)$ [17]. The edge gradient can be determined from these two images and the direction for each pixel is as follows:

$$
\begin{gathered}
\text { Edge Gradient }(G)=\sqrt{G_{x}{ }^{2}+G_{y}{ }^{2}} \\
\text { Angle }(\theta)=\tan ^{-1}\left(\frac{G_{x}}{G_{y}}\right)
\end{gathered}
$$

Gradient direction is always perpendicular to the edges. Hysteresis thresholding stage decides the real edges. After these steps, contrast enhancement is performed. These steps are followed by Histogram equalization for image enhancement. The basic idea behind this technique is mapping the intensity levels based on probability distribution of the input intensity levels.

Feature extraction is done after preprocessing to extract all the information for cataract detection and grading from the circular pupil region. The proposed detection algorithm is based on finding the accurate thresholds of texture feature parameters such as image intensity $(I)$, uniformity (U) standard deviation (s), to distinguish between healthy and abnormal eyes. In cataract eyes, the whitish color originates from the lens region so it can be easily concluded that cataract eyes have higher intensities than normal eyes. The equation to measure mean intensity $(\mathrm{m})$ is expressed as:

$$
m=\sum_{i=0}^{L-1}\left(\frac{I(i)}{N}\right)
$$

Here ' $m$ ' is average of intensity, ' $I$ ' is possible intensity, ' $N$ ' is number of pixel in an image, and ' $L$ ' is the value of possible intensity levels. Smooth and coarse textures are found in the eye. If clouding is present, a coarse texture will be observed which shows the presence of a cataract, with degree of severity depending upon the amount of clouding. Uniformity would be maximum when all gray 
levels have equal values. A healthy eye will show a smooth texture with higher value of uniformity in contrast to coarse texture. Uniformity can be calculated by the given equation:

$$
\mathrm{U}=\sum_{i=0}^{L-1}\left(\frac{H(i)}{N}\right)^{2}
$$

Here ' $U$ ' is measure of uniformity, ' $H$ ' is probability histogram of the intensity levels in an image region, and ' $N$ ' is the number of pixel in an image region, Let $i=0,1,2 \ldots, L-1$ be the corresponding histogram, and ' $L$ ' is the value of possible intensity. In terms of image processing, a low value of standard deviation indicates that the pixel value tends to be very close to the average value, whereas high value of standard deviation represents that the pixel values are broadly spread out over a large range of values [18]. The formula to calculate the standard deviation is given as:

$$
s=\sqrt{\frac{\sum\left(x_{i}-\bar{x}\right)^{2}}{r \times c-1}}
$$

Here, ' $x_{i}{ }^{\prime}$ is the individual pixel value of the input map considered by filter, ' $\bar{x}$ ' is the mean of the pixel values considered by the filter, ' $r$ ' is the size of the filter in rows and ' $c$ ' is the size of the filter in columns.

The calculation of standard deviation together with the mapping of diagnostic opinion of eye expert, adds robustness to the proposed algorithm as it helps in detecting almost exact pixel values as compared to the neighboring pixels.

Decision making requires a database for comparison with the characteristic values. For the purpose of the creation of a database, diagnostic opinion of an ophthalmologist regarding the presence of cataract and its severity was recorded for 50 patients under 300 lux light intensity conditions. An image was acquired by placing the camera (Sony Cybershot, Allahabad, India) at 1entimeters distance from the eye facing forwards with camera axis almost coinciding with pupil axis. The calculation of standard deviation together with the mapping of diagnostic opinion of an eye expert adds robustness to the proposed algorithm as it helps in detecting almost exact pixel values as compared to the neighboring pixels and mapping the values with the diagnostic opinion of ophthalmologist. Comments from ophthalmologist regarding healthy, mild and severe cases were recorded. Values of texture information (mean intensity, uniformity and standard deviation) have been calculated and recorded based on an eye expert's opinion for the classification between healthy and cataract affected eyes. Texture information and doctor's opinion were mapped so that a threshold for detection of cataract based on texture feature (mean intensity, uniformity and standard deviation) values can be derived. Texture information values were recorded for each case viz., healthy, mild cataract and severe cataract case as identified by a doctor. Later an individual mean value of these three cases, as per the doctor's opinion, were found using K-Means clustering technique [19]. For setting a threshold between a healthy eye and a cataract eye (both mild and severe cases), a range has been selected starting from the bottommost value recorded in a healthy eye case to the average value of K-Means cluster of two consecutive classes. The proposed system gives a decision based on this threshold value set for a normal eye and a cataract eye (both mild and severe cases together). For normal and cataract eyes, the above mentioned features have distinct values. For a healthy eye, the decision mean intensity threshold value was found to be less than 125.31 and more than 12.93 . Uniformity value for a healthy eye was found to be greater than 0.17 and standard deviation was found to be less than 1.59. For cataract suffering patient's eyes, two classes of decisions were made, namely mild and severe. For mild cataracts, mean intensity value was found to be between 125.3 and 134, uniformity value in between 0.09 and 0.11 . Standard deviation value was found to lie between 2.64 and 3.18. For severe cataract cases, mean intensity value was found to be more than 134, uniformity value less than 0.09 and standard deviation value greater than 3.18. These calculated thresholds for texture features are mentioned in Table 1. As explained in this section, the overall proposed algorithm can be summarized as: Image preprocessing which includes 
reading true color image, its conversion to grayscale image, contrast enhancement, noise removal using Gaussian filter, cropping the pupil area from the eye image, resizing the cropped image to $256 \times 256$ pixels size and histogram calculation. The next step is calculation of feature parameter (mean intensity, uniformity and standard deviation) values and decision making after comparing these feature parameter values from thresholds calculated in Table 1.

Table 1. Calculated texture information thresholds.

\begin{tabular}{cccc}
\hline \multirow{2}{*}{ Texture Information } & \multicolumn{3}{c}{ Doctor's Opinion } \\
\cline { 2 - 4 } & Healthy & Mild & Severe \\
\hline Mean Intensity $(I)$ & $12.93<I<125.31$ & $125.31<I<134$ & $I>134$ \\
Uniformity $(\mathrm{U})$ & $\mathrm{U}>0.17$ & $0.0927<\mathrm{U}<0.11$ & $\mathrm{U}<0.09$ \\
Standard Deviation $(s)$ & $s<1.59$ & $2.64<s<3.18$ & $s>3.18$ \\
\hline
\end{tabular}

Thresholding and database creation can be described by a block diagram in Figure 1.

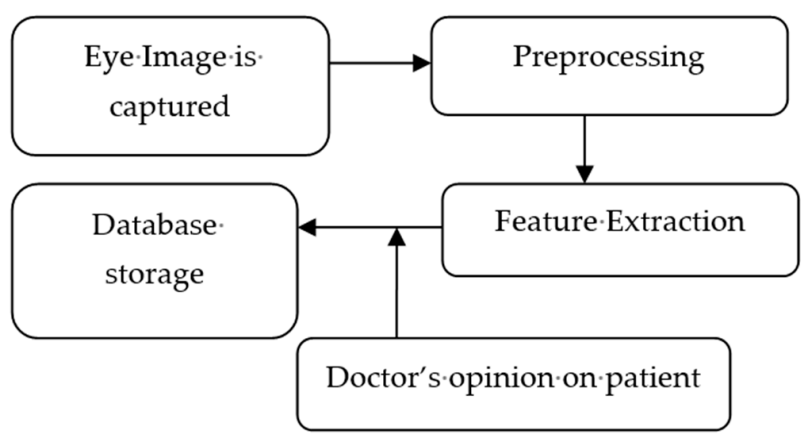

Figure 1. Block diagram of parameter thresholding and database creation.

\section{Experimental Outcomes and Diagnostic Validation}

Based on the observed texture information thresholds mapped with an eye expert's opinion, the proposed algorithm detects the presence of cataract from the colored digital image of an eye. A summary of the whole procedure can be described by a flowchart presented in Figure 2.

The simulations are performed using MATLAB R2013b. The images used for validation of current work are acquired from true color image database as well as those captured by Sony cyber shot digital camera during field trials. In the pre-processing of input image, various types of pre-processing techniques are applied which are described in previous section. The results of the pre-processed image are depicted in Figure 3a-e.

For a healthy eye, the pupil is very clear, i.e., no whiteness is present inside it. Mean intensity and standard deviation are quite low for normal eyes whereas uniformity is high. The histogram plot of a normal eye is shown in Figure 4. It can be seen that the different intensities are uniformly distributed in the histogram. An almost flat pattern is observed here in this histogram confirms that uniform distribution of pixels is present over the whole intensity range. Due to absence of any clouding, an almost flat intensity histogram is observed in Figure 4. In cataract suffering eyes, the illuminated pupil is brighter and has high intensity values present in the histogram and high standard deviation value, whereas uniformity is quite low. The histogram in Figure 5 represents a very high population of pixels with large intensity values and shows the presence of cataract. A non-uniform distribution of pixels at different intensities can be observed here in Figure 5.

Calculation of three texture information parameters (mean intensity, uniformity and standard deviation) together with diagnostic opinion of ophthalmologist adds robustness to this proposed algorithm. The decision made by the system regarding presence of cataract $(\mathrm{Y})$ and its absence $(\mathrm{N})$ is based upon comparison from set thresholds for mean intensity, uniformity and standard 
deviation matches which fit perfectly with the eye expert's opinion. During the training phase, it was observed that the decision regarding the presence of the cataract should be based upon at least two parameter values. Keeping this point in mind, a decision has been programmed in the proposed system. The results of the proposed system's trial conducted over 200 patients has been depicted briefly in Table 2 which shows different images with corresponding texture values, doctor's opinion and the decision given by the trained system regarding the presence of a cataract.

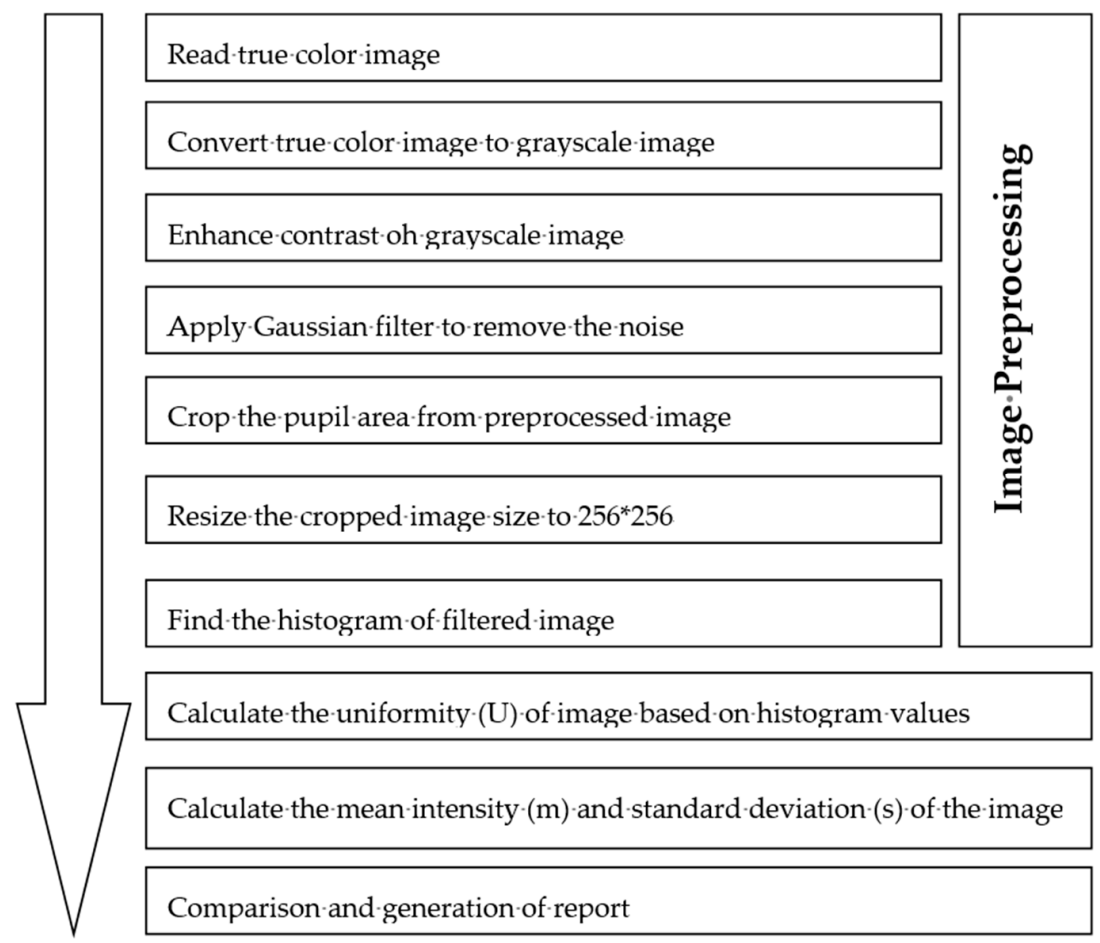

Figure 2. Flowchart of robust cataract detection algorithm.

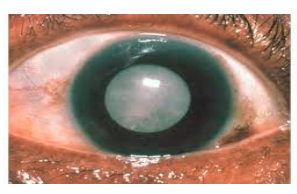

(a)

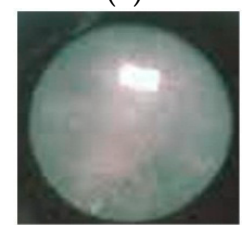

(c)

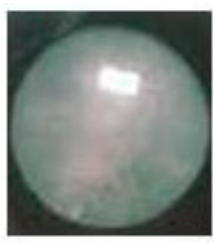

(b)

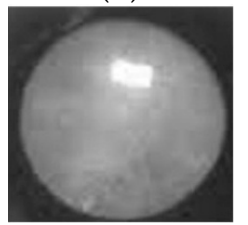

(d)

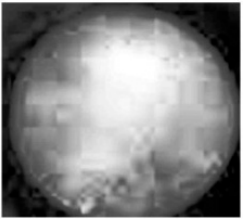

(e)

Figure 3. (a) Subject eye image; (b) Cropped pupil region; (c) Resized image $(256 \times 256)$; (d) Gray-scale image; (e) Contrast enhanced image. 


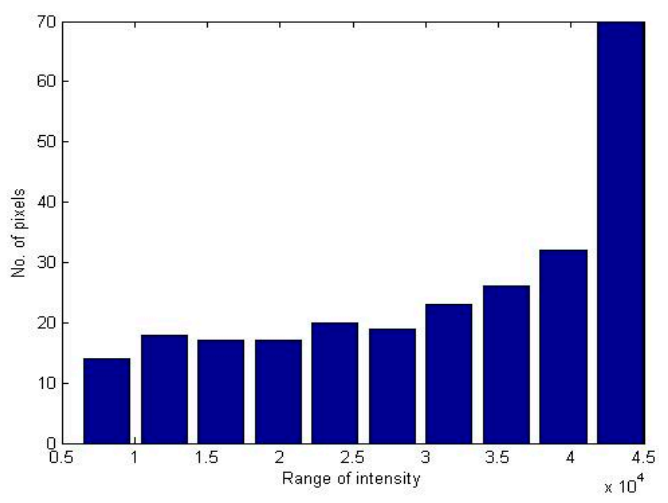

Figure 4. Histogram of healthy eye.

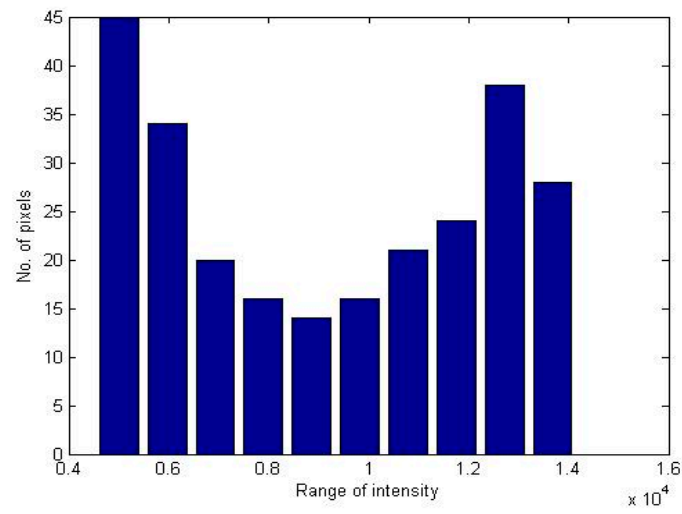

Figure 5. Histogram of cataract eye.

Table 2. Calculated texture information of patients and validation by Doctor.

\begin{tabular}{|c|c|c|c|c|c|}
\hline \multirow{2}{*}{ Image No. } & \multicolumn{3}{|c|}{ Calculated Texture Information } & \multirow{2}{*}{$\begin{array}{l}\text { Doctor's } \\
\text { Opinion }\end{array}$} & \multirow{2}{*}{$\begin{array}{c}\text { System's } \\
\text { Output (Y/N) }\end{array}$} \\
\hline & $m$ & $\mathbf{U}$ & $s$ & & \\
\hline 1. & 12.41 & 0.13 & 1.17 & Healthy & $\mathrm{N}$ \\
\hline 2. & 18.78 & 0.15 & 1.41 & Healthy & $\mathrm{N}$ \\
\hline 3. & 13.85 & 0.15 & 1.69 & Healthy & $\mathrm{N}$ \\
\hline 4. & 144.59 & 0.10 & 3.27 & Severe & $\mathrm{Y}$ \\
\hline 5. & 122.19 & 0.11 & 2.32 & Mild & $\mathrm{Y}$ \\
\hline 6. & 114.66 & 0.10 & 2.41 & Mild & $\mathrm{Y}$ \\
\hline 7. & 136.41 & 0.09 & 2.92 & Severe & $\mathrm{Y}$ \\
\hline 8. & 11.69 & 0.18 & 1.80 & Healthy & $\mathrm{N}$ \\
\hline 9. & 8.75 & 0.15 & 1.16 & Healthy & $\mathrm{N}$ \\
\hline 10. & 172.12 & 0.14 & 3.76 & Severe & $\mathrm{Y}$ \\
\hline 11. & 153.48 & 0.10 & 3.49 & Severe & $\mathrm{Y}$ \\
\hline 12. & 132.52 & 0.09 & 3.18 & Severe & $\mathrm{Y}$ \\
\hline 13. & 152.38 & 0.13 & 2.70 & Mild & $\mathrm{Y}$ \\
\hline 14. & 132.11 & 0.08 & 2.32 & Mild & Y \\
\hline 15. & 137.17 & 0.10 & 3.73 & Severe & $\mathrm{Y}$ \\
\hline
\end{tabular}

To add simplicity in application of our proposed algorithm, a graphic user interface (GUI) has been created in MATLAB to suggest that a user friendly system can be developed over other popular platforms, such as a webpage, android application or some other platform based application. It enables the proposed system to be hosted on a remote server with increased ease of access. The main aim to develop this system is to make the whole module user friendly and increase its accessibility. Figures 6 and 7 show the GUI with input image. When the pupil is not extracted automatically using 
Hough transform, a manual method of cropping has been kept in the proposed system for cropping the pupil area which is later diagnosed by our system for presence of a cataract. Creation of this GUI enables this robust cataract detection system to be accessed from a remote location. It enables the use of our proposed system in tele-ophthalmology application. A tele-ophthalmology model has been proposed in Figure 8, which shows a method to detect the cataract from a remote location by the help of our proposed algorithm and developed system. Here, a patient at a remote location in an infrastructure deprived environment such as a village as well as in an infrastructure-supported environment such as in a city, one can access this system and get the cataract diagnosis done. This will greatly help the people living in remote villages with no ophthalmologist or eye hospital at convenient locations. This will also aide the urban population by reducing the hassles of visiting an eye expert for cataract screening. As cataracts are one of the major causes of blindness amongst the ageing population, this system helps in timely and convenient diagnosis of someone from a remote location and hence finds its use in a tele-ophthalmology application.

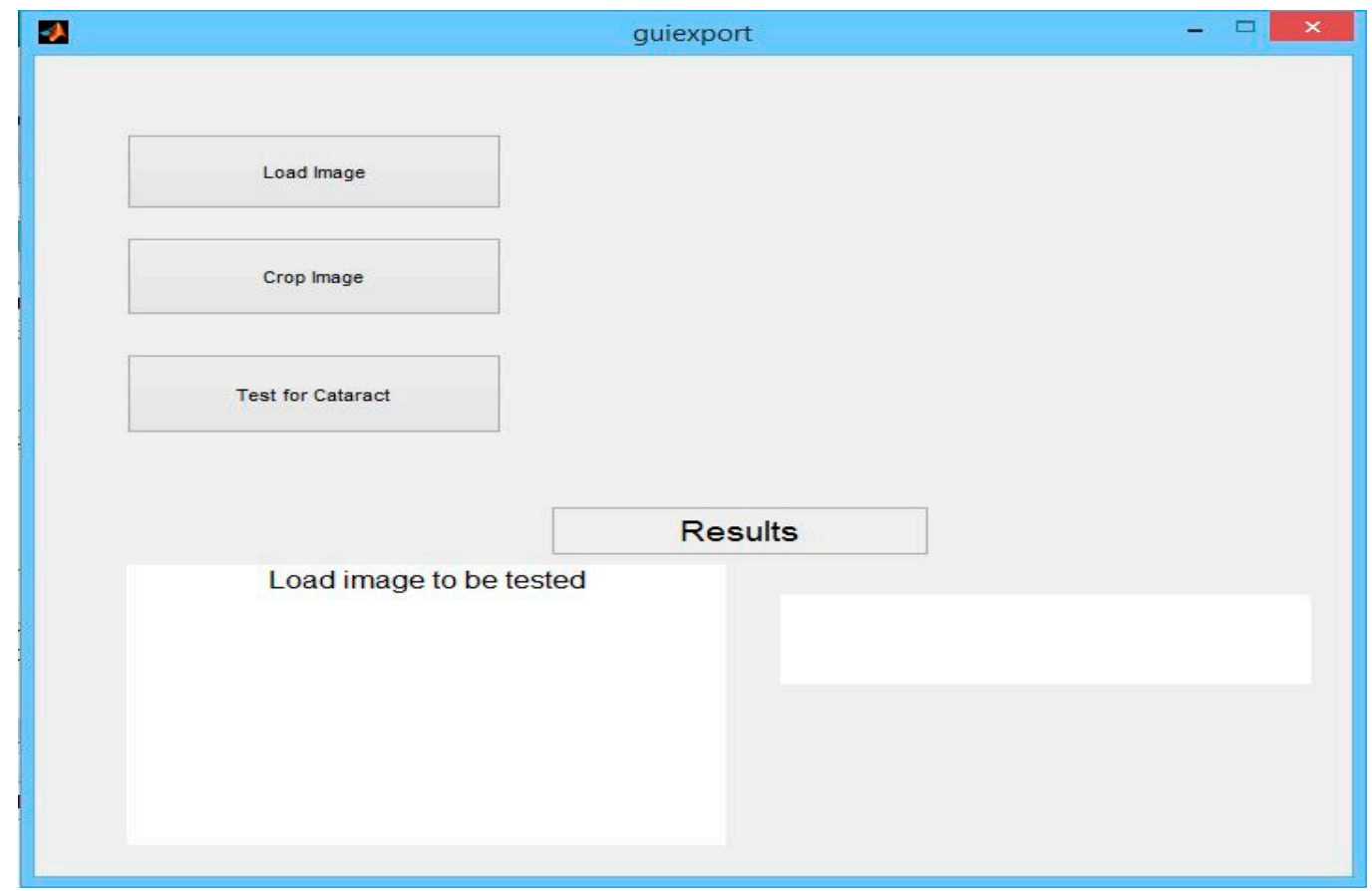

Figure 6. Initial input screen of the developed graphic user interface (GUI).

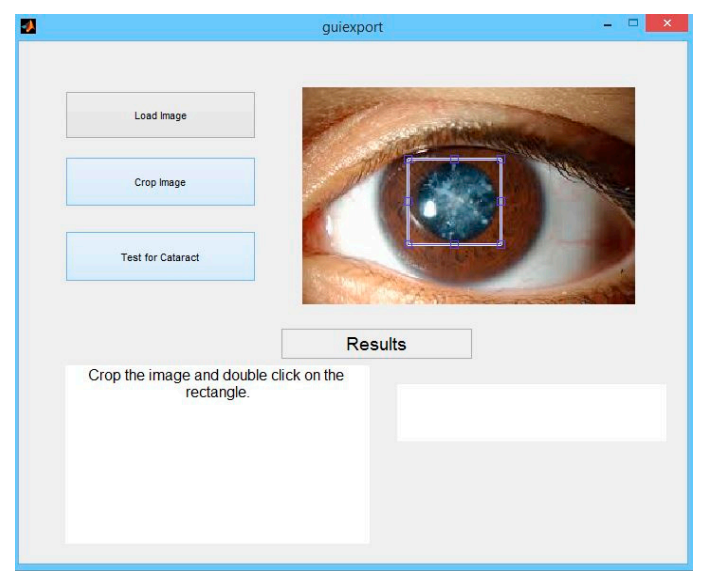

(a)

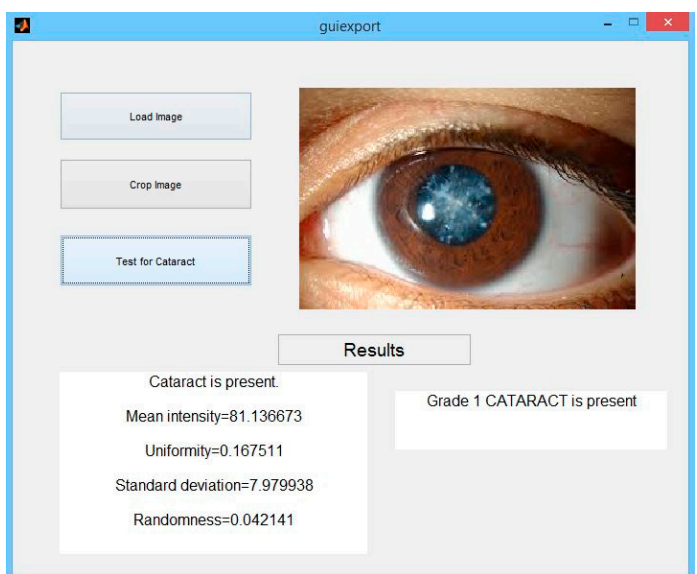

(d)

Figure 7. Cont. 


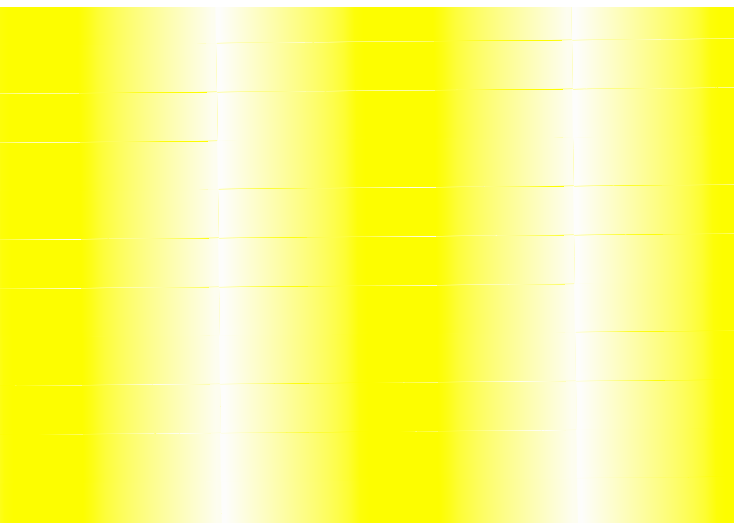

(b)

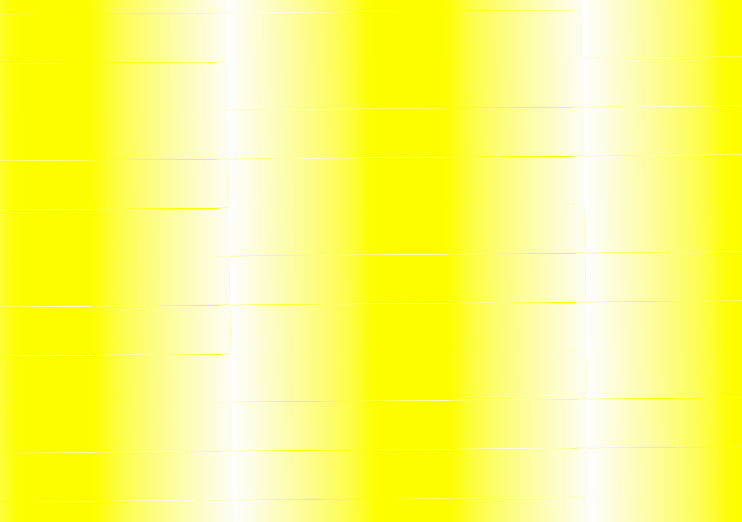

(c)

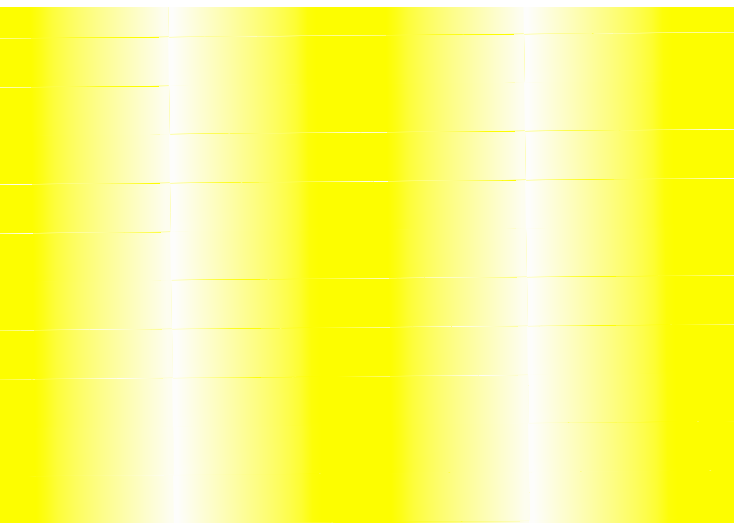

(e)

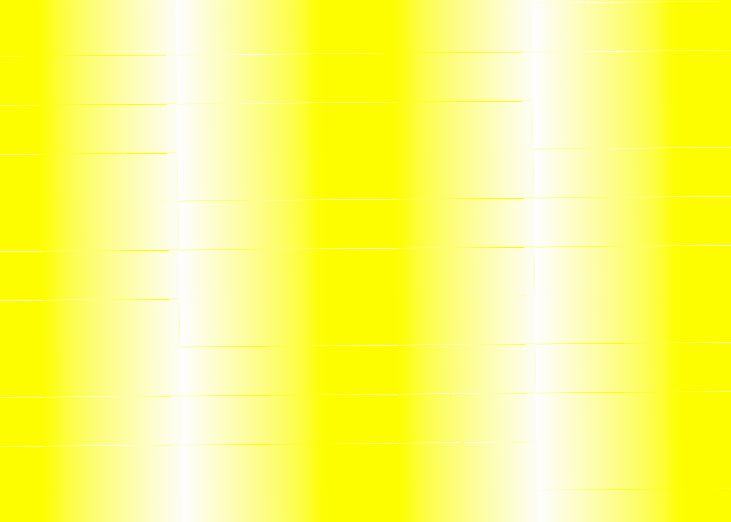

(f)

Figure 7. (a)-(c) An image is uploaded for cataract screening; (d)-(f): Corresponding result of the cataract screening software.

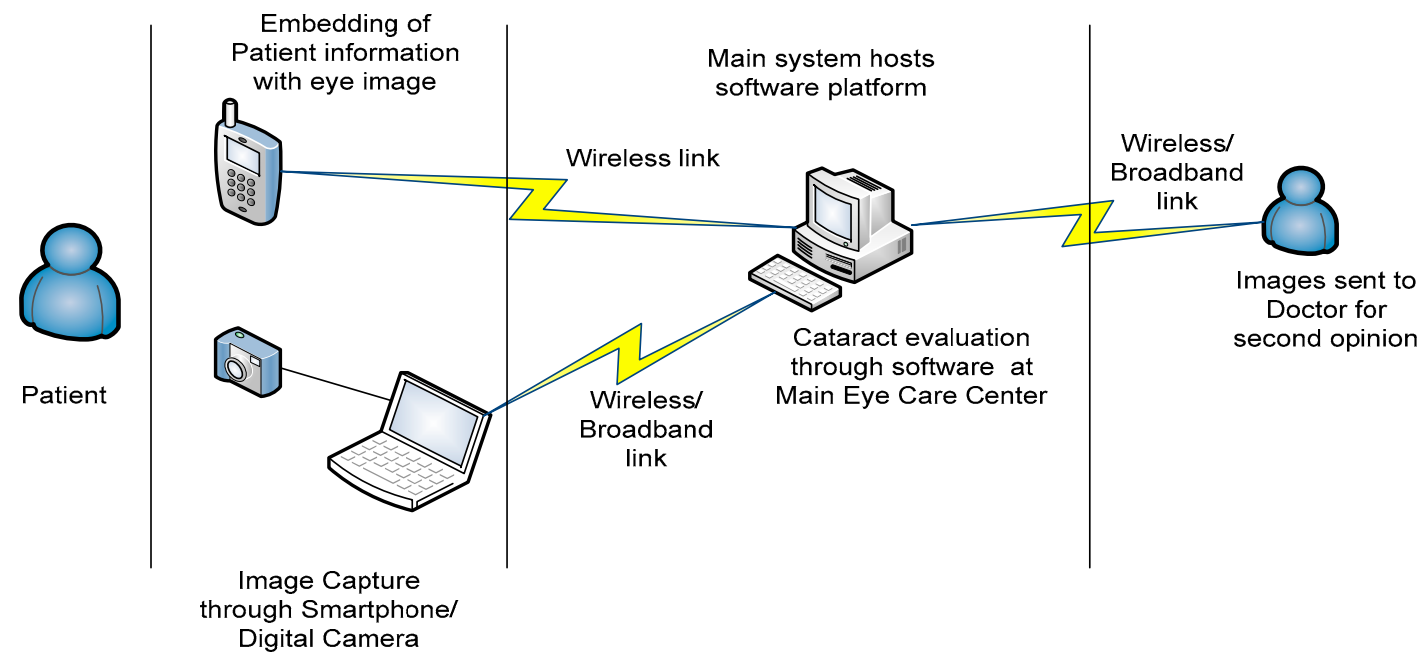

Figure 8. Network architecture of proposed tele-ophthalmology model.

In this tele-ophthalmology model, the proposed system will be placed as software in a server placed at a remote location. The patient will visit a primary eye screening center or access the system over a mobile computing device by registering on a mobile app for availing this cataract screening facility. The image will be uploaded by the technician or by a patient (in mobile app mode) and these images embedded with patient information will be sent to the server for primary cataract screening. A report will be generated by the system and sent to the patient and for secondary opinion to an eye 
expert for final decision. A common health monitoring web platform interfaced with suitable imaging modules will help doctors from remote locations to examine the patient and give a final decision. This way, a more reliable diagnosis can be made.

\section{Conclusions}

This paper presented a texture information based automated algorithm for detection of cataracts from a digital eye image of adult human subjects. Experiments were performed on true color images obtained from a low cost compact digital camera. It was observed that inclusion of standard deviation parameter together with mean intensity and uniformity features added robustness to the decision made by the algorithm which was validated by the ophthalmologist. The proposed method gave very encouraging results with close to $98 \%$ accuracy but this needed to be further tested on larger dataset for more accurate detection of a mild or an early stage cataract. The proposed algorithm detects presence of cataract by reading texture information from circular pupil of adult human subjects. It is not tested on child subjects, also it cannot detect cataract in the child subjects with those suffering from coloboma, i.e., the child subjects who have non circular pupils. A GUI has been created in MATLAB for adding simplicity to the operation of proposed system and a network architecture has been proposed for its implementation in telemedicine application. Future work includes fine tuning of the threshold parameters for cataract detection by considering a large number of patients and corresponding ophthalmologists' decisions. Intelligent computing methods such as SVM or machine learning can be used for improved results. Efforts are being made to remove the flash spot of the camera during preprocessing and evaluate its effect. The algorithm can further be extended for predicting severity information in terms of cataract grading. Different wireless networks will be evaluated for the required quality of service parameter requirements at different layers for the proposed tele-ophthalmology architecture.

Acknowledgments: We thank the administration, doctors and staff of Life Line Hospital Barauni, Bihar (India) for extending support in conducting field trial of the proposed robust and low cost automated cataract detection system. We are grateful to M.K. Singh, Professor, Dept. of Ophthalmology, Institute of Medical Sciences BHU, India for providing sufficient training data and giving his diagnostic opinion for validation of the system. We thank the learned reviewers and editor for constructive criticisms and suggesting the valuable improvements in the current manuscript.

Author Contributions: B.K. conceived the idea of portable cataract detection sytem, S.P. defined the parameters for testing, managed the implementation, conceived telemedicine architecture and conducted field trials. S.P. prepared the current manuscript and B.K helped in editing the manuscript.

Conflicts of Interest: The authors declare no conflict of interest.

\section{References}

1. Cost of Vision Problems. Available online: http://costofvision.preventblindness.org/home/introduction (accessed on 20 April 2016).

2. Visual Impairment and Blindness. Available online: http://www.who.int/mediacentre/factsheets/fs282/ en/ (accessed on 20 April 2016).

3. Gary, B.; Taylor, H. Cataract Blindness-Challenges for 21st century. Bull. World Health Organ. 2001. Available online: http:/ / www.who.int/bulletin/archives/79(3)249.pdf (accessed on 30 April 2016).

4. Comas, O.; Cotin, S.; Duriez, C. A shell model for real time simulation of intra-ocular implant deployment. In Proceedings of the International Symposium on Biomedical Simulation, Phoenix, AZ, USA, 23-24 January 2010; pp. 160-170.

5. Supriyanti, R.; Habe, H.; Kidode, M.; Nagata, S. A simple and robust method to screen cataract using specular reflection appearance. In Proceedings of the Medical Imaging International Conference of International Society for Optics and Photonics (SPIE), San Diego, CA, USA, 17 March 2008. [CrossRef]

6. Supriyanti, R.; Habe, H.; Kidode, M.; Nagata, S. Cataract Screening by Specular Reflection and Texture Analysis. In Proceedings of the Systemics and Informatics World Network (SIWN 2009), Leipzig, Germany, 23-25 March 2009. 
7. Supriyanti, R.; Habe, H.; Kidode, M.; Nagata, S. Compact cataract screening system: Design and practical data acquisition. In Proceedings of the International Conference on Instrumentation, Communication, Information Technology and Biomedical Engineering (ICICI-BME), Bandung, Indonesia, 23-25 November 2009. [CrossRef]

8. Shen, H.; Hao, H.; Wei, L.; Wang, Z. An image based classification method for cataract. In Proceedings of the International Symposium on Computer Science and Computational Technology, Shanghai, China, 20-22 December 2008. [CrossRef]

9. Yang, M.; Yang, J.-J.; Zhang, Q.; Niu, Y.; Li, J. Classification of retinal image for automatic cataract detection. In Proceedings of the e-Health Networking, Applications \& Services (Healthcom), Lisbon, Portugal, 9-12 October 2013. [CrossRef]

10. Nayak, J. Automated classification of normal, cataract and post cataract optical eye images using SVM classifier. In Proceedings of the World Congress on Engineering and Computer Science, San Francisco, CA, USA, 23-25 October 2013; pp. 542-545.

11. Li, H.; Lim, J.H.; Liu, J.; Mitchell, P.; Tan, A.G.; Wang, J.J.; Wong, T.Y. A Computer-Aided Diagnosis System of Nuclear Cataract. IEEE Trans. Biomed. Eng. 2010, 57, 1690-1698. [PubMed]

12. Caixinha, M.; Velte, E.; Santos, M.; Santos, J.B. New approach for objective cataract classification based on ultrasound techniques using multiclass svm classifiers. In Proceedings of the IEEE International Ultrasonics Symposium (IUS), Chicago, IL, USA, 3-6 September 2014. [CrossRef]

13. Caxinha, M.; Velte, E.; Santos, M.; Perdigão, F.; Amaro, J.; Gomes, M.; Santos, J. Automatic cataract classification based on ultrasound technique using machine learning: A comparative study. Phys. Procedia 2015, 70, 1221-1224. [CrossRef]

14. Srivastava, R.; Gao, X.; Yin, F.; Wong, D.W.; Liu, J.; Cheung, C.Y.; Wong, T.Y. Automatic nuclear cataract grading using image gradients. J. Med. Imaging 2014, 1, 014502. [CrossRef] [PubMed]

15. Yang, J.-J.; Li, J.; Shen, R.; Zeng, Y.; He, J.; Bi, J.; Li, Y.; Zhang, Q.; Peng, L.; Wang, Q. Exploiting ensemble learning for automatic cataract detection and grading. Comput. Methods Progr. Biomed. 2016, 124, 45-57. [CrossRef] [PubMed]

16. Rashmi; Kumar, M.; Saxena, R. Algorithm and Technique on Various Edge Detection: A Survey. Signal Image Process. 2013, 4, 65. [CrossRef]

17. Deng, G.; Cahill, L.W. An adaptive Gaussian filter for noise reduction and edge detection. In Proceedings of the 1993 IEEE Conference Record, Nuclear Science Symposium and Medical Imaging Conference, San Francisco, CA, USA, 31 October-6 November 1993. [CrossRef]

18. Sepasian, M.; Balachandran, W.; Mares, C. Image enhancement for fingerprint minutiae based algorithm using CLAHE, standard deviation analysis and sliding neighbourhood. In Proceedings of the World congress on Engineering and Computer Science (WCECS 2008), San Francisco, CA, USA, 22-24 October 2008.

19. Kanungo, T.; Mount, D.M.; Netanyahu, N.S.; Piatko, C.D.; Silverman, R.; Wu, A.Y. An efficient k-means clustering algorithm: Analysis and implementation. In Proceedings of the Sixteenth ACM Symposoium on Computational Geometry, Hong Kong, China, 12-14 June 2000; pp. 100-109.

(C) 2016 by the authors; licensee MDPI, Basel, Switzerland. This article is an open access article distributed under the terms and conditions of the Creative Commons Attribution (CC-BY) license (http://creativecommons.org/licenses/by/4.0/). 